\title{
Evolutionary perspectives on overeating and overweight. Introduction to the special section of Appetite
}

It has been estimated that the epidemic of overweight and obesity has now reached more than 1.1 billion people worldwide (World Health Organization, 1998). The rapid increase in the number of people being overweight has received considerable attention in the past decade with a particular emphasis on epidemiology and the disastrous health consequences of obesity. In addition, considerable research efforts have been spent on designing behavioral, pharmacological and surgical interventions to treat or prevent obesity (Wadden, Brownell, \& Foster, 2002). In spite of these efforts, it is still poorly understood which factors have contributed to the sharp increase of obesity in the past 30 years. Some authors have pointed to the rapid economic and social changes in modern society (Chou, Grossman, \& Saffer, 2004), resulting in copious amounts of palatable high energy food being readily available while at the same time reducing physical daily demands to very low levels - coined as 'the toxic food environment' (Horgen \& Brownell, 2002). The fact that modern living conditions offer individuals ample opportunity to eat as much as they want, any time any place, is relevant to a discussion on obesity but it does not explain why people would engage in this type of behavior, knowing that it makes them fat in the short run and compromises their health in the long run.

This state of affairs may be the reason that an evolutionary explanation of the current epidemic of obesity is becoming increasingly popular. Evolutionary accounts of overweight in modern society emphasize that humans have evolved to eat more than necessary to meet their immediate nutritional needs as a buffer against future periods of food scarcity (Pinel, Assanand, \& Lehman, 2000). The biological systems involved in human weight regulation thus promote levels of consumption that maintain the energy resources of the body well above the levels required to meet immediate needs, so that excess energy can be stored in the body to protect from future famine. Whereas this regulation system was once adaptive in an environment characterized by scarce food supplies, it has become maladaptive in the modern environment characterized by an abundance of food. Indeed, the eating regulation system seems to be simply overpowered by the food-replete environments in which we now live: "Genes load the gun, the environment pulls the trigger" (Bray, 1998).

Adopting an evolutionary point of view is compelling as it provides a rather straightforward account of a once adaptive mechanism that has become maladaptive in a society that is characterized by the abundance of food. Obesity thus seems an excellent case for testing evolutionary principles. However, this area has received almost no attention by evolutionary psychologists - even though good arguments can be made for the claim that food selection has been the single most important force in human evolution (Rozin, 2000). In 2004, we organized an invitational conference at Utrecht University examining the potential of an evolutionary view on obesity. This special section presents a selection of papers, all highlighting a particular aspect of an evolutionary view of modern obesity.

In the first paper, Lieberman summarizes some of the well-known facts about the modern obesogenic environment, illustrating that current conditions related to obtaining food differ dramatically from the food environment of our ancestors. Applying optimal foraging theory, Lieberman demonstrates that it takes hardly any effort to obtain food in modern society. Moreover, she shows that the food industry is, intentionally or unintentionally, very successful in exploiting visual food cues, which interfere with adequate monitoring of food consumption, making humans extremely vulnerable to overconsumption.

The next paper by Mela discusses the role of pleasure in eating as the alleged responsible factor for developing overweight. In contrast with the popular evolutionary notion that pleasure is the sole force in regulating eating behavior, he challenges the idea that obesity is the result of a particular liking for palatable foods, leading to excess intake. He argues that a disturbed motivation to obtain food is a more likely candidate for explaining overweight as the wanting of foods is severely compromised by modern living conditions emphasizing the availability of food.

De Graaf examines the role of another seemingly typical feature of modern eating patterns, the frequent ingestion of high-energy snacks. His review of the literature demonstrates that, as expected, most individuals do not 
compensate for the consumption of snacks but it also shows that the evidence for the role of snacks in developing overweight is mixed. De Graaf suggests that it may not be the irregular consumption of foods per se that is responsible for overweight, but rather the nature of snacks: liquid snacks, be they soft drinks or yoghurt-like beverages, are likely to make the difference as liquid calories have not been a regular ingredient of mammal diets since the beginning of human evolution.

In their paper on the role of self-control in overweight, Van den Bos and De Ridder challenge the common evolutionary view that food consumption is driven by the availability of food only. They argue that the biological systems involved in regulating eating behavior are attenuated by intuitive decisions about the trade-off between the immediate pleasure of eating and long-term benefits concerning food supplies (and maybe also health considerations). As choosing for immediate benefits may be an adaptive response under uncertain conditions, it appears that modern individuals continue to behave as if food supplies are still uncertain, resulting in a dysfunctional pattern of overeating.

Finally, Polivy and Herman address what seems to be a typical modern feature of eating behavior-the voluntary restriction of food intake. They argue that dieting may have adaptive benefits, but only when there is limited availability of food supplies - suggesting that dieting may not be a 'modern' behavior after all. When there is no shortage of foods, as in modern conditions, there is no point in dieting; as demonstrated in the difficulty of dieting in the presence of food. They conclude that diet may be the worst response to an ecology of plenty, resulting in maladaptive eating behaviors.

Together, these papers demonstrate the potential of an evolutionary perspective on overeating and obesity, while at the same time providing directions for future research to examine the ways in which the biological heritage of our ancestors may have shaped dysfunctional responses to an eating environment that has no precedents in the history of human evolution.

\section{Acknowledgment}

Preparation of this special section of Appetite on 'Evolutionary Approaches to Overeating and Overweight' was supported by Grant 051-14-013 of the Dutch Organization of Scientific Research, Evolution and Behaviour Program.

\section{References}

Bray, G. A. (1998). Contemporary diagnosis and management of obesity. Newton, PA: Handbooks in Health Care.

Chou, S. Y., Grossman, M., \& Saffer, H. (2004). An economic analysis of adult obesity: Results form the behavioral risk factor surveillance system. Journal of Health Economics, 23, 565-587.

Horgen, K. B., \& Brownell, K. D. (2002). Confronting the toxic environment: Environmental, public health actions in a world crisis. In T. A. Wadden, \& A. J. Stunkard (Eds.), Handbook of obesity treatment (pp. 95-106). New York: Guilford.

Pinel, J. P. J., Assanand, S., \& Lehman, D. R. (2000). Hunger, eating, and ill health. American Psychologist, 55, 1105-1116.

Rozin, P. (2000). Evolution and adaptation in the understanding of behavior, culture, and mind. American Behavioral Scientist, 43, 970-986.

Wadden, T. A., Brownell, K. D., \& Foster, G. D. (2002). Obesity: Responding to the global epidemic. Journal of Consulting and Clinical Psychology, 70, 510-525.

World Health Organization. (1998). Obesity: Preventing and managing the global epidemic. Geneva, Switzerland.

Denise de Ridder

Department of Health Psychology, Utrecht University, PO Box 80140, 3508 TC Utrecht, The Netherlands

E-mail address: D.deRidder@fss.uu.nl

Ruud van den Bos

Department of Ethology and Welfare, Utrecht University,

The Netherlands 